\title{
Prevalence of human immunodeficiency virus among tuberculosis patients in Khartoum state
}

\begin{abstract}
Introduction: Pulmonary Tuberculosis (TB) and human immunodeficiency virus (HIV) have been closely connected since the appearance of acquired immunodeficiency syndrome (AIDS). HIV infection among active TB cases provides real challenges in diagnosis and treatment of TB. This is descriptive cross-sectional study conducted at Abu-anja Teaching Hospital; Khartoum state in period from October 2018to March 2019.The objective was to determine the prevalence of Human Immunodeficiency Virus infection among tuberculosis patients.
\end{abstract}

Method: A total of 90 venous blood samples were collected from the study participants, serum was obtained by centrifugation at $(3000 \mathrm{rpm})$ for 5 minutes. The levels of HIV antibody titer were determined by using sandwich ELISA Assay.

Results: The studies revealed that out of the 90 participants of the tuberculosis patients, $5(5.6 \%)$ were HIV positive, and the HIV prevalence among male patients were significantly higher than female patients.

Conclusion: The study concluded that there is high prevalence of HIV among tuberculosis patients in Khartoum state, capital of Sudan (5.6\%) comparing with $(5 \%)$ at which the WHO recommended intensified intervention to address TB-HIV co-infection critical in any strategy that aims to reach those most in need.

Keywords: prevalence, human immunodeficiency virus, tuberculosis, Khartoum state
Volume 8 Issue 3 - 2020

\author{
Waseem Sameer Kwami,' Abass Ahmed Ali \\ Ahmed,' Mosab Nouraldein Mohammed \\ Hamad $^{2}$ \\ 'Department of Microbiology, Faculty of Medical Laboratory \\ Sciences, University of Shendi, Sudan \\ 2Phylum of Medical Parasitology, Department of Medical \\ Laboratory Science, Elsheikh Abdallah Elbadri University, Sudan \\ Correspondence: Mosab Nouraldein Mohammed Hamad, \\ Phylum of Medical Parasitology, Department of Medical \\ Laboratory Science, Elsheikh Abdallah Elbadri University, Sudan, \\ Emailmusab.noor3@gmail.com
}

Received: June 04, 2020 | Published: June 30, 2020

\section{Introduction}

HIV infected people are in a great danger for death by tuberculosis and TB/HIV co-infection has been approved to reduce the impact of directly observed therapy (DOT) strategy treatment of TB. Mycobacterium tuberculosis is the causative agent of tuberculosis that most often affect the lungs. The probability of developing TB for those infected with Mycobacterium tuberculosis is 5-15\%, however, that problem is substantially increased in persons who are Immunocompromised such as those infected with Human Immunodeficiency Virus (HIV). ${ }^{1}$ Mycobacterium tuberculosis infects 1.7 Billion people around the world. Out of these, 8 million new cases are affected by active tuberculosis. In total, TB is responsible for around three million fatalities each year. ${ }^{2}$

Human Immunodeficiency Virus (HIV) infects the immune system; specially helper $\mathrm{T}$ lymphocytes leading to progressive loss of cellular and eventually all immune components. This leads to the development of acquired immunodeficiency syndrome (AIDS) eventually which is indicated by susceptibility to opportunistic infections and virulent pathogens and AIDS-related neoplasms. ${ }^{3}$ The disease progress is accelerated for both pathogens in the context of coinfection, with higher mortality than infection of either HIV of MTB. Three-hundred-thousand cases died from HIV-associated TB in 2017, with approximately one million new cases of TB among HIV positive cases, most of whom (72\%) live in Africa. ${ }^{4}$

TB has a worldwide distribution attacking the population of every continent. However; the majority of TB cases take place in South-
East Asia and Western Pacific; which account for $62 \%$. Africa comes second with $25 \%$ of global TB cases. ${ }^{1}$ In 2017 , eighty-seven per cent of incident TB cases occurred in the 30 high TB burden countries. 8 countries accounted for two thirds of the new TB cases: India, China, Indonesia, the Philippines, Pakistan, Nigeria, Bangladesh and South Africa. ${ }^{1}$

HIV/TB co-infection management is a complicated endeavor, with a huge treatment failure and resistance to antimicrobials. More than a million people live with HIV/AIDS now typically affecting the poor, the homeless, intravenous (IV) drug users, alcoholics, the elderly or those with debilitating illnesses. ${ }^{5}$ Tuberculosis (TB) is a leading cause for morbidity and mortality and the control of HIV and tuberculosis epidemics is a real challenge facing sub Saharan African countries. ${ }^{4}$ The rate of Tuberculosis-related mortality in Sudan is estimated at 25.0 per 100000 population. A total of 20181 detected tuberculosis cases were indicated in 2013, of which 5980 (30\%) were new sputum smear-positive cases. Association of tuberculosis with HIV is the one of the most frightened challenges to the national tuberculosis program in Sudan. ${ }^{5}$

Sudan is an endemic area of tuberculosis, the first case of HIV and AIDS in Sudan was reported in 1986, however there are few available published data in Sudan concerning TB /HIV co -infection. ${ }^{6}$ Early detection of HIV among TB patients is very important for preventive and control purposes; it provides an opportunity to implement the prophylactic therapy and antiretroviral treatment that reduces the morbidities and mortality. Also support the treatment strategy of TBAIDS co-infection. ${ }^{6}$ 


\section{Method}

\section{Specimen collection}

A total of 90 venous blood samples were collected in plain container, from pulmonary tuberculosis patients who participated in this study; the serum was obtained by centrifugation at $(3000 \mathrm{rpm})$ for 5 minutes.

\section{Procedure of ELISA technique}

The reagents were allowed to reach room temperature $\left(18-30^{\circ} \mathrm{C}\right)$ for 25 minutes, and the wash buffer concentration was checked for the presence of salt crystals then diluted 20 times with distilled water. ${ }^{7}$ The strips were set in strip-holder and numbered with sufficient number of wells included three negative control (B1, C1, D1), three positive control (E1, F1, G1), and one Blank (A1). ${ }^{7} 20 \mu 1$ of conjugate reagent (biotinylated anti-HIV p24antibodies) was added into each well except the blank.100 $\mu$ l of positive control, negative control, and specimen were added into their respective wells by separate disposable pipette tip. ${ }^{7}$

The plate was covered by its cover and incubated for 60 minutes at $37^{\circ} \mathrm{C}$ in thermostat-controlled water tank. After the end of the incubation, the plate cover was removed and discarded. And the wells were washed, each well 5 times with diluted Wash buffer. After the final washing cycle, the plate was turned down onto blotting paper and taped. And any remain liquids was removed. $100 \mu$ l of HRPConjugate Reagent was added into each well except the blank, and mixed gently. ${ }^{7}$ The plate was covered with its cover and incubated for 30 minutes at $37^{\circ} \mathrm{C}$. After the end of the incubation, the plate cover was removed and discarded. And the wells were washed, each well 5 times with diluted Wash buffer. After the final washing cycle, the plate was turned down onto blotting paper and taped. And any remain liquids was removed. ${ }^{7}$

$50 \mu 1$ of chromogen A and $50 \mu \mathrm{l}$ chromogen B solution were dispensed into each well included the blank, and the plate was covered by its cover and mixed gently by tapping. The plate was incubated at for $37^{\circ} \mathrm{C} 15$ minutes and the light were avoided. Blue color was produced in positive control and HIV1/2 positive for antibodies sample wells. ${ }^{7}$ The plate cover was removed and discarded. 50 $\mu$ l Stop Solution was added into each well by multichannel pipette and mixed gently. Intensive yellow color was developed in positive control and HIV1/2 positive for antibodies sample wells.?

\section{Reading and interpretation of the results}

The plate reader was calibrated with the blank well and the absorbance was read at $450 \mathrm{~nm}$ within 15 minutes after the reaction stop. Cut-off- value (C.O) was calculated and the results evaluated. ${ }^{7}$ The presence or absence of antibody for HIV1/2 was determined by comparing the absorbance measured for each sample with the cut-offvalue. ${ }^{7}$ Samples give absorbance more than cut-off-value indicate that theHIV1/2 antibodies are present (positive result), while the samples give absorbance less than cut-off-value indicate that the HIV1/2 antibodies are absence (negative result). ${ }^{7}$

\section{Results}

In this study a total of 90 participants were included the majority of them $60(73.3 \%)$ were males and only $30(26.7 \%)$ were females. The age of study participants ranged from 18 to 65 years, 60 of them were less than 40 years old and only 30 were more than 40 years old.
In this study out of 90 study participants $67(74.4 \%)$ were follow up cases and 23(25.6\%) were new cases. Out of the 90 participants of the tuberculosis patients, $5(5.6 \%)$ were HIV positive, and $85(84.4 \%)$ were HIV negative (Table 1).

Table I Frequency of HIV infection among study population

\begin{tabular}{lll}
\hline Study population & Frequency & Percentage \\
\hline HIV Positive & 5 & $5.60 \%$ \\
HIV Negative & 85 & $94.40 \%$ \\
Total & 90 & $100 \%$ \\
\hline
\end{tabular}

In this study out of all 66-tuberculosis male patient $7.5 \%$ ( 5 cases) showed HIV positive result and all the 24 female participants showed HIV negative result $(\mathrm{p}$-value $=0.000)$ (Table 2$)$.

Table 2 Frequency of HIV infection among the gender

\begin{tabular}{llll}
\hline Sex & Frequency & Percentage & P-value \\
\hline Males $(\mathrm{n}=66)$ & 5 & $7.50 \%$ & 0 \\
Females $(\mathrm{n}=24)$ & 0 & $0 \%$ & \\
\hline
\end{tabular}

Out of 23 new tuberculosis cases only 1(4.3\%) showed HIV positive result, and out of 57 tuberculosis follow up cases $4(5.9 \%)$ showed HIV positive result ( $p$-value $=0.0001)$ (Table 3$)$.

Table 3 Frequency of HIV infection among the different TB Status group

\begin{tabular}{llll}
\hline TB Status & Frequency & Percentage & P-value \\
\hline New cases $(n=23)$ & I & $4.30 \%$ & \\
Follow up $(n=67)$ & 4 & $5.90 \%$ & 0.0001 \\
\hline
\end{tabular}

In this study only 2 cases (3.3\%) out of 60 tuberculosis patients who were less than 40 years old were HIV positive and 3 cases (10\%) out of 30 tuberculosis patients who were above 40 years old were HIV positive (p-value $=0.01)$ (Table 4).

Table 4 Frequency of HIV infection among the age group

\begin{tabular}{llll}
\hline Age group & Frequency & Percentage & P-value \\
\hline Less than 40Years $(n=60)$ & 2 & $3.30 \%$ & \\
Above40Years $(n=30)$ & 3 & $10 \%$ & 0.01 \\
\hline
\end{tabular}

\section{Discussion}

This study involved 90 Sudanese TB patients and subjected to detection the present of anti-HIV. The study found that out of $90 \mathrm{~TB}$ patients, the Prevalence of HIV among TB patients was found (5.6\%), the prevalence was lower than those reported from Metema hospital, Northwest Ethiopia by (Tarekegne et al 2016) in which the prevalence was $(20.1 \%)$ and also the prevalence was lower when compared to similar study from Kasala city in Sudan by (Abdallah et al.,2011) which was $(18.3 \%)$.This low prevalence may be attributed to small sample size applied in this study when compared with the sample size of thosestudies. ${ }^{8,6}$

The prevalence of HIV among TB patients in this study, was found to be differed with different gender. In which the male TB patients showed significant statistical increase in HIV positive cases $(7.5 \%)$ than female TB patients $(0 \%)$. This finding was inconsistent with study conducted in in Port Harcourt, Nigeria by (Erhabor et al., 
2010) who observed a higher HIV prevalence rate among females $(80 \%)$ as compared to males $(20 \%)$. This difference may explain by involvement of male in outdoor activities involving risky practices (like extramarital sexual activities, intravenous drug abuse, alcohol consumption) which could have put them at increased risk for HIV. ${ }^{9}$

In this study there was statistically significant high HIV prevalence rate among TB patients of age above 40years than those who were less than 40Years ( $\mathrm{p}$ value $=0.01$ ). This finding is indifference with previous study conducted by (Haar et al., 2006) who found that the highest HIV seropositive rate among patients aged between 30 to 39 years. The high rate of HIV among those who are more than 40 years may be due to low immune system efficiency due to aging. ${ }^{10}$ In this study the rate of HIV cases among TB follow up cases (5.9\%) were significantly higher than new TB cases $(4.3 \%)$. The low rate among new cases may be due to immune system level which still strong in the primary level of TB.

\section{Conclusion}

The study concluded that the seroprevalence of HIV among tuberculosis patients in Khartoum state was $5.6 \%$ and the prevalence among male tuberculosis patients was higher than female patients, and individuals of age more than 40 years old revealed a higher prevalence of HIV rate.

\section{Acknowledgments}

None.

\section{Conflicts of interest}

Authors declare that there is no conflict of interest.

\section{References}

1. Liyan W, Wei L, Lu W, et al. HIV Prevalence among Pulmonary Tuberculosis Patients in Guangxi, China. Acquir Immune Defic Syndr. 2010;53(Suppl 1):S61-S65.

2. Tille PM. Bailey, Scott's. Diagnostic microbiology. 14th ed. St. Louis, Missouri: Elsevier; 2017.

3. Brooks G, Carroll KC, Butel J, et al. Medical Microbiology. 26th ed. Blacklick: McGraw-Hill Publishing; 2012.

4. Blumberg HM, Burman WJ, Chaisson RE, et al. American Thoracic Society/Centers for Disease Control and Prevention/Infectious Diseases Society of America: treatment of TB. Am J Respir Crit Care Med. 2003;167(4):603-662.

5. John M, Menezes CN, Chita G, et al. High tuberculosis and HIV coinfection rate, Johannesburg. Emerg Infect Dis. 2007;13(5):795-796.

6. Abdallah TM, Siddig MF, Ali AA. Seroprevalence of HIV infection among tuberculosis patients in Kassala, eastern Sudan. Journal of AIDS and HIV Research. 2011;3(8):161-163.

7. www.Fortressdiagnostics.com

8. Daniel T, Muhabaw J, Tadesse A, et al. Prevalence of human immunodeficiencyvirus infection in a cohort of tuberculosis patients at Metema Hospital, Northwest Ethiopia: a 3 years retrospective study. BMC Res Notes. 2016;9:192.

9. Erhabor $\mathrm{O}$, Jeremiah $\mathrm{Z}$, Adias $\mathrm{T}$, et al. The prevalence of human immunodeficiency virus infection among TB patients in Port Harcourt Nigeria. HIV/AIDS-Research and Palliative Care. 2010:2:1-5.

10. Haar CH, Cobelens FGJ, Kalisvaart NA, et al. HIV prevalence among TB patients in the Netherlands, 1993-2001: trends and risk factors. Int J Tuberc Lung Dis. 2006;10(7):768-774. 